\title{
Laser Machining of Chosen Materials
}

Prof. Imrich Lukovics ${ }^{1)}$, MSc., Ph.D., Martina Malachova ${ }^{2)}$, MSc., M.A.

${ }^{1)}$ Department of Production Engineering, Faculty of Technology, Tomas Bata University in Zlín, Nad Stráněmi 4511, 760 05, Zlín, Czech Republic, lukovics@ft.utb.cz

${ }^{2)}$ Technical University of Ostrava, Faculty of Mechanical Engineering, 17.listopadu 15/2172, Ostrava-Poruba, 708 33,

Czech Republic, malachova@fhs.utb.cz

A paper deals with technological applications of laser. It evaluates the influence of design and technological conditions on output parameters of cutting process and also presents relative laser machinability of polymeric and metallic materials; in addition, it shows possible evaluation of structural change of metals and it gives mathematical model for determination laser cutting quality functions based on results of the experimental research. The temperature distribution has been derived. Results of plastic materials and hard-to-machining metals machinability are shown. Finally, surface quality evaluation after exposure to concentrated light energy and quantification of material microhardness are described.

Keywords: laser machining, technological conditions, simulation.

\section{References}

[1] HALAŠKA, P., MAŇAS, M., SLOBODIAN, P (2002). Laser cutting optimalilization of the polymeric plates and films.In.: 42nd Science Week Laser Science and Applications, University OF Aleppo, Syria, p.80.

[2] HRABČÁKOVÁ, I., FABIAN, S. (2006). Kvantifikace vlivu technologických parametrů na parametry kvality při řezání laserem. Strojírenská technologie XI. ISSN 12114162, s. 16-21.

[3] HUGEL, H. et al.(1992). Strahlwerkzeug Laser. B.B. Teubner, Stuttgart.

[4] LUKOVICS, I., MAŇAS, M. (1996). Možnosti využití laseru a paseru pro zpracování polymerů. Plasty a kaučuk roč. $33, \check{c} .8$, s. 228 .

[5] LUKOVICS, I., MALACHOVÁ, M. (2007). Use of Laser in Engineering. Manufacturing Technology VII December 07. ISSN 1213-2489, s. 26-31

[6] LUKOVICS, I., SÝKOROVÁ, L. (2003). Laser Machinability of Polymers and Difficult-to-machine Materials. Manufacturing Technology III. ISSN 12132489, s. 20-26.

[7] MAŇKOVÁ, I. (2000). Progresívné technologie, Vienala Košice.

[8] RADOVANOVIČ, M. R. (2004). Mathematical Model for Severance Energy by CO2 Laser Cutting of Mild Steel. Strojírenská technologie IX záŕí 2004. Nr.3. ISSN 1211-4162, s. 20-26

[9] SÝKOROVÁ, L. (2001). Výzkum obrábění nekovových materiálů laserem. Disertační práce.VUT FSI Brno.

[10] SÝKOROVÁ, L., Lukovics, I. (2008). Optimalizace vstupních parametrů a ekonomické aspekty laserového popisování knižních vazeb. Strojírenská technologie XIII/2. ISSN 12114162, s. 9-14.

[11] SÝKOROVÁ, L., Shejbalová, D., Lukovics, I. (2005). Laser Workability and Surface Quality Characteristic of Plastics. Manufacturing Technology V. ISSN 1213248-9, s. 25-31.

[12] SÝKOROVá, L., Malachová, M. (2012). Laser Machining and Temperature Field Simulation Using COSMOS / M Software. Manufacturing Technology. Nr. 12. ISSN 12132489, s. 113-117.

[13] ŠUBA O., SYKOROVA L., MALACHOVA M., SAMEK D. (2010). Modelling of Transient Thermal Stress in Layered Walls. Manufacturing Technology X December 2010. Nr. 10. ISSN 1213-2489, s. 16-19

[14] TURŇOVÁ, Z. - LOŠÁK, G. (2004). Safety of Laser Technologies. In.: New Ways in Manufacturing Technologies 2004. TU Košice, FVT Prešov, 2004, p. 584 - 589, ISBN 80-8073-136-5.

[15] WU, J; DAY, D ; GU, M (2011). Polymerci optofluidic Fabry-Perot sensor by direkt laser machining and hot embossing. Applied Optics, Vol. 50, pp. 1843-1849, ISSN: 1559-128X

Paper number: M201209 